\title{
La virtualización de servidores como una herramienta para la optimización de recursos.
}

Server virtualization as a tool for the optimization of resources.

Diego Jácome Segovia. ${ }^{1}$, Jessica Núñez Núñez. ${ }^{2}$, Velasteguí López Efraín. ${ }^{3,}$ Milton Navas Moya. ${ }^{4} \&$ Paco Vásquez Carrera. ${ }^{5}$

Recibido: 15-12-2017 / Revisado: 08-02-2018 Aceptado: 05-03-2018/ Publicado: 01-04-2018

\begin{abstract}
.
DOI: https://doi.org/10.33262/cienciadigital.v2i2.93

In a world that is becoming more and more technified and linked to the dependence of new technologies, it is evident that most organizations are focused on implementing initiatives that allow them to be more efficient and competitive, but without neglecting the optimization of both economic resources as technological and in the same way to apply policies that benefit the environment, there are technologies that are born as those alternatives to achieve these goals, one of them is Green Computing or Green Technologies from which comes the virtualization of servers that undoubtedly is a topic that is currently in full development, so companies and institutions today are looking for mechanisms to introduce these new technologies into their technological environments, with the purpose to optimize resources and take advantage of them in a more efficient way. This research is oriented in the comparative analysis between the different existing virtualization tools, so we have taken as reference the most important such as VMware 10 and Citrix XenServer 6.5, as these technologies help the process of server virtualization integrating all services, these tools will offer the best stability and appropriate management of technological resources.
\end{abstract}

\footnotetext{
1 Universidad Técnica de Cotopaxi Ext. La Maná, Cotopaxi, Ecuador, diego.jacome@utc.edu.ec

2 Universidad Técnica de Cotopaxi, Cotopaxi, Ecuador jessicanuñez@gmail.com

3Universidad Técnica de Cotopaxi, Cotopaxi, Ecuador,luis.velastegui7838@utc.edu.ec

${ }^{4}$ Universidad de las Fuerzas Armadas Ext. Latacunga, Ecuador, mpnavas@espe.edu.ec

${ }^{5}$ Universidad Técnica de Cotopaxi Ext. La Maná, Cotopaxi, Ecuador, paco.vasquez@utc.edu.ec
} 
Keywords: Virtualization, Green Computing, Green Technologies, Resource Optimization, VMware 10 and Citrix XenServer 6.5

\section{Resumen.}

En un mundo cada vez más tecnificado y acoplado a la dependencia de las nuevas tecnologías, es evidente que la mayor parte de organizaciones se ven abocadas en aplicar iniciativas que le permitan ser más eficientes y competitivas pero sin dejar de lado la optimización de recursos tanto económicos como tecnológicos y de idéntica manera en aplicar políticas que beneficien al medio ambiente, existen tecnologías que nacen como aquellas alternativas para lograr estos objetivos, una de ellas es el Green Computing o Tecnologías Verdes de la cual se desprende la virtualización de servidores que sin duda alguna es un tema que en los actuales momentos se encuentra en pleno desarrollo, es así que las empresas e instituciones hoy en día están buscando mecanismos para introducir estas nuevas tecnologías en sus entornos tecnológicos, con el propósito optimizar los recursos y aprovecharlos de una manera más eficiente. La presente investigación está orientada en el análisis comparativo entre las diferentes herramientas de virtualización existentes, es así que se ha tomado como referencia las más importantes como son VMware 10 y Citrix XenServer 6.5, ya que estas tecnologías ayudan al proceso de virtualización de servidores integrando todos los servicios, estas herramientas ofrecerán la mejor estabilidad y el manejo adecuado de los recursos tecnológicos.

Palabras Claves: Virtualización, Green Computing, Tecnologías Verdes, Optimización de recursos, VMware 10 y Citrix XenServer 6.5

\section{Introducción.}

En los últimos años el avance tecnológico sin duda alguna que ha desatado una imperiosa necesidad de que todas las organizaciones estén en el nivel de adquirirlas para poder seguir compitiendo en el amplio mundo de los negocios. En tal sentido las nuevas alternativas en las cuales confían las organizaciones está en el ámbito de modernizarse tecnológicamente y reducir costos operativos, es así que muchas instituciones han optado por implementar procesos que generen una mayor rentabilidad y rendimiento a la organización tomando en cuenta lo que hoy en día se conoce como la virtualización de servidores que son procesos aplicables con el propósito de reducir costos La Virtualización es la tecnología que permite a las empresas consolidar su infraestructura de TI para poder así a lograr un mayor aprovechamiento de los recursos. La Virtualización es una capa abstracta que desacopla el hardware físico del sistema operativo para brindar una mayor flexibilidad y utilización de los recursos de TI. El computador físico comparte recursos para crear las máquinas virtuales (VM). La planificación del CPU puede compartir CPU para crear la apariencia de que los usuarios 
tienen sus propios procesadores. La operación periférica simultánea en línea y el sistema de archivos ofrecen lectoras de tarjetas virtuales e impresoras virtuales. Una terminal normal de usuario con tiempo compartido proporciona la función de la consola del operador de la máquina virtual. Una máquina virtual ofrece ciertas ventajas tales como una protección completa de diversos recursos del sistema. Cada máquina virtual está aislada por completo de las demás, por lo que no hay problemas de seguridad. Por otro lado, no se comparten recursos directamente. Además, una MV "Máquina Virtual" proporciona un vehículo perfecto para la investigación y desarrollo de sistemas operativos. Otra gran ventaja al utilizar una máquina virtual es que proporciona un medio para soluciona la compatibilidad entre sistemas. Una de las primeras iniciativas hacia la informática verde en los Estados Unidos fue el programa voluntario de etiquetado conocido como Energy Star. Fue concebido por la Agencia de Protección Ambiental (EPA) en 1992 para promover la eficiencia energética en el hardware de todo tipo. La etiqueta Energy Star se convirtió en algo común, especialmente en el ordenador portátil y pantalla. Programas similares se han adoptado en Europa y Asia.

\section{Métodos.}

\section{Green Computing.}

"Green Computing" o Informática verde generalmente se refiere a la utilización eficiente de los recursos en la informática, en relación con la minimización del impacto ambiental, y la maximización de la viabilidad económica, similar a cualquier otro problema de consumo de recursos del objetivo de la informática verde es a: (I) el uso de materiales menos peligrosos (II) maximizar la eficiencia de todo el uso de recursos en los sistemas de computación durante su vida, y (III) reutilizar la mayor cantidad de recursos posible y desechar lo que no se puede reciclar de forma responsable. (KHAN, 2013).

\section{Cloud Computing o Computación en la nube.}

Agrupa las tecnologías, servicios y aplicaciónes que son similares a las de internet y las convierte en utilidades de autoservicio. El uso de la palabra "cloud" se refiere a dos conceptos esenciales Abstracción y Virtualización. (JOYANES, 2012)

Abstracción. La computación en la nube abstrae los detalles de la implementación del sistema de los usuarios y desarrolladores. Las aplicaciónes se ejecutan en sistemas fisicos que no estan especificados, los datos se almacenan en posiciones que son desconocidas, la administración de sistemas esta externalizada a otros y el acceso por parte de los usuarios.

\section{Virtualización}

Abtracción de los recursos de un computador mediante una máquina virtual (VM, Virtual Machine) o hipervisor que es una capa de abstracción entre el hardware de la máquina física (host) y el sistema operativo de la máquina virtual (client,guest) y proporciona un medio para crear una version virtual de un dispositivo o recurso, como un servidor, un 
dispositivo de almacenamiento, una red o, incluso un sistema operativo, donde se dicide el recurso en uno o más entornos de ejecución. (GOYANES, 2012).

\section{Implementación de la Virtualización.}

Existen diferentes formas de implementar la virtualización. Dos de los enfoques líderes en utilización son: Virtualización Completa (Full virtualización) y Paravirtualización (Paravirtualization).

\section{Virtualización Completa.}

La Virtualización completa está diseñada para proporcionar abstracción total del sistema físico fundamental y crea un sistema virtual completo en que se puede ejecutar el sistema operativo huésped (guest). No se requiere ninguna modificación en el SO o aplicación huésped. El SO o aplicación cliente no aprecia el entorno virtualizado, de modo que se puede ejecutar en la máquina virtual (VM) como si ella fuera un sistema físico. Este enfoque puede ser ventajoso ya que facilita el desarrollo completo del software del hardware. La virtualización completa puede relacionar la migración de aplicaciones y cargas de trabajo entre diferentes sistemas físicos y también ayuda a hacer este enfoque muy seguro. NIST (National Institute of Standards and Technology), en la virtualización completa, uno o más sistemas operativos y las aplicaciónes que contienen se ejecutan en la parte superior (top) del hardware virtual. Cada instancia de un sistema operativo (SO) y sus aplicaciónes se ejecutan en una máquina virtual (VM) independiente, llamada sistema operativo huésped (guest). La virtualización completa permite ejecutar sistemas operativos huésped (guest) sin tener que modificarlos, sobre un sistema anfitrion (host) utilizando entre ambos un hypervisor o virtual Machine Monitor (VMM) que permite compartir el hardware real y controla el flujo de instrucciones en el sistema operativo huésped y el hardware físico tal como la CPU, el almacenamiento en disco, memoria y tarjetas de interfaces de red.

\section{Ventajas de la Virtualización.}

- Algunas ventajas de la virtualización son:

- La virtualización ofrece ahorro sustancial mediante la reducción de costes de energía, instalaciones, mano de obra, espacio y dinero.

- Se pueden Virtualizar distintos recursos como sistemas operativos, servidores, almacenamiento, aplicaciones.

- Facilidad para hacer cambios.

- Utilizar sistemas operativos y aplicaciones que ya no tienen soporte sin interferir con otras aplicaciones.

- Los programadores y desarrolladores de aplicaciones pueden probar sus programas en cualquier sistema operativo.

\section{Algunos inconvenientes de la Virtualización.}

Falta de confianza en la nueva tecnología 
Es necesario formar a personas para que puedan utilizar esta tecnología

Cambiar a un sistema virtualizado es complejo y es necesaria una planificación. (NIÑO, 2011).

\section{Categorías de Virtualización.}

Los dos tipos más extendidos en los centros de datos son: virtualización de servidores y virtualización de almacenamiento. Sin embargo, se pueden considerar otros enfoques como sub conjuntos de estos o como modelos independientes. Sun Microsystems (hoy Oracle) clasificaba sus tecnologías de virtualización en:

- Virtualización de Servidores

- Virtualización de almacenamiento

- Virtualización de escritorio

\section{Virtualización de Servidores .}

Es un método de particionar o dividir un servidor físico en multiples servidores de modo que cada uno de ellos tenga la aparariencia y capacidades como si fuera una máquina dedicada. De este modo los servidores individuales se dividen en dominios independientes, aislados unos de otros para asegurar que no haya interferencias entre un dominio y otro. Cada dominio puede contener una o más máquinas virtuales. El aislamiento del dominio asegura a los clientes que los diferentes sistemas no tienen forma de interferir con la integridad de los otros sistemas restantes.

\section{Virtualización de Almacenamiento.}

Otro método de virtualización que utilizan las organizaciones es la virtualización del almacenamiento. Esta tendencia se manifiesta en una red de almacenamiento de área (SAN Storage Area Network).

\section{Virtualización del Escritorio.}

La virtualización del escritorio del cliente permite que en cada técnica, el PC se controle desde el centro de datos (no desde el escritorio). En la practica la virtualización entraña el uso del software para emular un entorno informático con otro ordenador. La virtualización cliente implica la emulación de un PC completo en el software de un servidor de un centro de datos y la visualización del interfaz de usuario en una terminal gráfica.

\section{Máquinas Virtuales.}

Es un software que emula un ordenador, es decir, es como tener un ordenador dentro de otro ordenador, pero funcionando de forma virtual, es decir, en realiad no se tiene un ordenador dentro de otro ordenador, ya que eso es imposible, pero lo que hace una máquina virtual es simularlo. Por medio de este software es posible instalar sistemas operativos adicionales (denominado sistemas invitado), dentro de otro sistema operativo (denominado sistema anfitrión), cada uno con su propio ambiente. En realidad, las máquinas virtuales no 
tiene procesador, memoria, conexión de red, puertos, discos duros, unicamente lo simulan. (RAYA J. M., 2010)

\section{Tipos de Máquina Virtuales.}

Hipervisor. El elemento encargado de realizar la virtualización en el host se denomina hipervisor o VMM (Virtual Machine Monitor, o monitor de máquina virtual). Hay dos tipos de hipervisores.

Nativo: el hipervisor es el host y se ejecuta directamente sobre el hardware del equipo.

Alojado: el hipervisor se ejecuta como una aplicación más sobre el sistema operativo del host. La interacción con los recursos del equipo se hara a traves de dicho sistema operativo. (GALLEGO, 2014).

Citrix XenServer. Utiliza el hipervisor Xen Project como un componente principal de su arquitectura para proporcionar una abstracción estable y elástica de la infraestructura subyacente. La tecnología de Xen Project es ampliamente reconocida como la plataforma de virtualización más veloz y más segura de la industria.

Sistemas operativos soportados. A diferencia de los Jails de FreeBSD o de los Zones/Containers de Solaris, que solo funcionan con una copia aislada del sistema mismo, la paravirtualización permite ejecutar varios sistemas operativos diferentes y de forma simultanea. A continuación se puede apreciar la lista de sistemas operativos soportados por ambas versiones, destacando que XenServer cuenta con "presets" pre configurados para brindar el máximo desempeño en algunos de ellos.

- Microsoft Windows 64-bit, Incluyendo Server 2012, 2008, 2003, y Windows 7

- Microsoft Windows 32-bit, Incluyendo Server 2012, 2008, 2003, Windows 2000, XP 7

- Linux 64-bit, Incluyendo RHEL, CentOS, Oracle, Novell SUSE, Debian y Ubuntu

- Linux 32-bit, Incluyendo RHEL, CentOS, Oracle, Novell SUSE, Debian y Ubuntu

- Solaris 10.

Aspectos Importantes de Citrix XenServer. Compatibilidad ampliada con virtualización de GPU.

Con mejores arquitectónicas, XenServer ahora es compatible con las últimas tarjetas NVIDIA GRID y más GPU físicos por host, lo que incrementa la capacidad de ampliación de las sesiones vGPU en un 50\% (desde VM 64 a VM 96). La plataforma de administración de Hypervisor XenServer es capaz de ampliarse efectivamente a medida que crece el hardware.

\section{Compatibilidad mejorada para la virtualización de servidores empresariales.}

XenServer 6.5 introduce de nuevo el balance de carga de trabajo (WLB) y es compatible con la generación de informes y monitorización de la carga de trabajo. Esto da a los 
administradores del sistema una visión profunda del rendimiento del sistema, lo que permite la optimización de la infraestructura y mover dinámicamente las cargas de trabajo basándose en las cargas de redes, almacenamiento y computación. XenServer puede incluso colocar cargas de trabajo automáticamente basándose en datos históricos. La funcionalidad de generación de informes es particularmente útil para que los administradores entiendan la salud general de los sistemas, así como las métricas como la capacidad del sistema.

\section{Mejoras a las redes, almacenamiento y disponibilidad.}

Un número de actualizaciones de arquitectura han incrementado significativamente el rendimiento de la red, hasta un $800 \%$ en algunos casos, ahora compatible con descarga de receptor genérico (GRO), la habilidad de ahorrar recursos de CPU kernel dom0 y rendimiento de huésped IPv6 mejorado. Grant Mapping se ha incrementado un 100\% en rendimiento agregado de múltiples máquinas virtuales. Sus mejoras de rendimiento de red permiten ejecutar más máquinas virtuales por host. El rendimiento del almacenamiento también se ha incrementado en más de un $100 \%$ con compatibilidad con almacenamiento en caché de lectura en memoria y tapdisk3, y se ha reducido el IOPS en las redes de almacenamiento.

\section{Arquitectura de próxima generación de 64 bits completos.}

XenServer ha tenido siempre un corazón de 64 bits, estando basado en el Hypervisor Xen (un proyecto de la fundación Linux). También tienen una larga compatibilidad tanto con sistemas operativos de invitados de 32 y 64 bits, aunque el dominio de control (Dom0) anteriormente era de 32 bits. En esta versión, XenServerv6.5 ahora se entrega con dominio de control de 64 bits, que tiene un profundo impacto en el host. No hay limitaciones de sistema operativo de 32 bits, el dominio de control XenServer es ahora capaz de acceder directamente a mucha más memoria (RAM) y tratar más adaptadores PCI que nunca. Ambas mejoras incrementan la capacidad de ampliación y el rendimiento de todo el sistema y permiten que XenServer sea compatible con la última generación de hardware de servidores de 64 bits de OEM.

\section{Mantenimiento y administración mejorada.}

Las mejoras de mantenimiento y administración de XenServer 6.5 permiten a los administradores instalar un conjunto de parches con un número mínimo de reinicios de host. Además, las versiones de controlador de dispositivos se han desacoplado de las versiones construidas en kernel cuando es posible a fin de evitar que se revierta a controladores de dispositivos obsoletos. Donde sea requerido, los paquetes de servicio futuros ahora incluirán los controladores de dispositivos nuevos y el proceso de notificación Hotfix se ha mejorado. (CITRIX, 2013). 


\section{Optimización de recursos tecnológicos.}

La optimización de recursos tecnológicos consiste en la maximización del patrimonio de la entidad utilizando de la manera más eficiente los recursos de la empresa tiene o a los que puede acceder. La estrategia de recursos tecnológicos tiene como objetivo principal la capitalización del patrimonio tecnológico de la entidad, en particular en los sectores en los que la empresa no está presente, adquiriendo competencias tecnológicas complementarias. (BERMEJO, 2014).

\section{Recursos tecnológicos.}

- Un recurso es un medio de cualquier tipo que permite satisfacer las distintas necesidades que se puedan presentar.

- Uno de los principales recursos con los que cuenta el hombre son los recursos tecnológicos.

- Un recurso tecnológico, por lo tanto, es un medio que se vale de la tecnología para cumplir con su propósito.

- Los recursos tecnológicos pueden ser tangibles (como una computadora, una impresora u otra máquina) o intangibles (un sistema de aplicación virtual). (ROJAS, 2013).

\section{Ventajas.}

Contribuye a reducir los costes. Cuando más numerosa sean las ocasiones de aplicar las tecnologías y los conocimientos, mayores serán las experiencia adquirida y el control de los costes.

Incremento el número de innovaciones puestas en el mercado. Cuanto más variadas sean las situaciones en las que se aplique un Know.how, tanto mayores serán las posibilidades de innovar; posibilidades que son el resultado de afrontar exigencias nuevas con la experiencia acumulada.

Aumenta la flexibilidad. Desde el momento en que el núcleo de las competencias tecnológicas se convierte en el eje central de la estrategia de desarrollo empresarial, la compañía estar mejor preparada técnica y comercialmente, sobre todo psicológica y culturalmente, para afrontar los necesarios cambios de actualidad.

Moviliza a la empresa. A diferencia de las estrategias de productos y de mercados los cuales cambian y desaparecen, la estrategia de recursos moviliza a la empresa alrededor de un proyecto a largo plazo, coordinando las capacidades individuales y colectivas alrededor de su núcleo tecnológico. 


\section{Tipos de recursos.}

\section{Recursos humanos.}

La administración de recursos humanos (personal) es el proceso administrativo aplicado al acrecentamiento y conservación del esfuerzo, las experiencias, la salud, los conocimientos, las habilidades, de los miembros de la organización, en beneficio del individuo, de la propia organización y del país en general.

Así mismo puede considerarse como el proceso de ayudar a los empleados a alcanzar un nivel de desempeño y una calidad de conducta personal y social que cubra sus necesidades. El propósito de la administración de recursos humanos es mejorar las contribuciones productivas del personal a la organización, de manera que sean responsables desde un punto de vista estratégico, ético y social. Este es el principio rector del estudio y la práctica de la administración de recursos humanos. Los gerentes y los departamentos de recursos humanos logran sus metas cuando se proponen fines claros y cuantificables. Estos objetivos equivalen a parámetros que permiten evaluar las acciones que se llevan a cabo. En ocasiones los objetivos se consignan por escrito, en documentos cuidadosamente preparados. En otras no se expresan de manera explícita, sino que forman parte de la "cultura de la organización".

Objetivos corporativos: Es necesario reconocer el hecho fundamental de que la administración de recursos humanos tiene como objetivo básico contribuir al éxito de la empresa o corporación. Incluso en las empresas en que se organiza un departamento formal de recursos humanos para apoyar la labor de la gerencia, cada uno de los supervisores y gerentes continúa siendo responsable del desempeño de los integrantes de sus equipos de trabajo respectivos. La función del departamento consiste en contribuir al éxito de estos supervisores y gerentes. La administración de recursos humanos no es un fin en sí mismo, es sólo una manera de apoyar la labor de los dirigentes.

Objetivos funcionales: Mantener la contribución del departamento de recursos humanos a un nivel apropiado a las necesidades de la organización es una prioridad absoluta. Cuando la administración de personal no se adecua a las necesidades de la organización se desperdician recursos de todo tipo. La compañía puede determinar, por ejemplo, el nivel adecuado de equilibrio que debe existir entre el número de integrantes del departamento de recursos humanos y el total del personal.

Objetivos sociales: El departamento de recursos humanos debe responder ética y socialmente a los desafíos que presenta la sociedad en general y reducir al máximo las tensiones o demandas negativas que la sociedad pueda ejercer sobre la organización. Cuando las organizaciones no utilizan sus recursos para el beneficio de la sociedad dentro de un marco ético, pueden verse afectadas por restricciones. 
Objetivos personales: El departamento de recursos humanos necesita tener presente que cada uno de los integrantes de la organización aspira a lograr ciertas metas personales legítimas. En la medida en que el logro de estas metas contribuye al objetivo común de alcanzar las metas de la organización, el departamento de recursos humanos reconoce que una de sus funciones es apoyar las aspiraciones de quienes componen la empresa. De no ser este el caso, la productividad de los empleados puede descender o también es factible que aumente la tasa de rotación. La negativa de la empresa a capacitar al personal podría conducir a una seria frustración de los objetivos personales de sus integrantes.

\section{Recursos Materiales.}

Estos resultan fundamentales para el éxito o fracaso de una gestión administrativa, lo básico en su administración es lograr el equilibrio en su utilización. Tan negativo es para la empresa en su escasez como su abundancia. Cualquiera de las dos situaciones resulta antieconómica; de ahí que la administración de recursos materiales haya cobrado tanta importancia actualmente.

\section{La administración de recursos materiales consiste en:}

Obtener oportunamente, en el lugar preciso, en las mejores condiciones de costo, y en la cantidad y calidad requerida, los bienes y servicios para cada unidad orgánica de la empresa de que se trate, con el propósito de que se ejecuten las tareas y de elevar la eficiencia en las operaciones.

\section{Recursos financieros.}

\section{La administración de recursos financieros:}

Supone un control presupuestal y significa llevar a cabo toda la función de tesorería (ingresos y egresos). Es decir, todas las salidas o entradas de efectivo deben estar previamente controladas por el presupuesto. Para estar en condiciones de evitar fallas y de aplicar correcciones oportunamente, corresponde al área financiera realizar los registros contables necesarios. Estos registros contables deben corresponder al presupuesto efectuándose por unidad organizacional.

\section{La administración financiera consiste en:}

Obtener oportunamente y en las mejores condiciones de costo, recursos financieros para cada unidad orgánica de la empresa que se trate, con el propósito de que se ejecuten las tareas, se eleve la eficiencia en las operaciones y se satisfagan los intereses de quienes reciben los bienes o servicios. (SANCHEZ, 2008) 


\section{Descripción de la guía de usuario para virtualización}

La guía de usuario es uno de los aspectos importantes al momento de implementar un proyecto de virtualización, ya que se deben tomar en cuenta varias aristas, como la metodología a seguir para ello se proponen los siguientes elementos:

- Análisis de la situación actual de los laboratorios en relación al consumo de recursos.

- Establecer los equipos tecnológicos con sus características.

- Esquematizar gráficamente el entorno virtual a implementar

- Determinar el área (entornos, sistemas operativos)

- Seleccionar las herramientas de virtualización adecuadas.

- Establecer los servidores físicos a virtualizar.

- Pruebas

- Implementación

\section{Análisis de la situación actual de los laboratorios.}

Tomando en cuenta la realidad de la infraestructura tecnológica de la Universidad Técnica de Cotopaxi Extensión La Maná, se hace necesario establecer un estudio del uso de los recursos antes de aplicar la propuesta planteada, es así que como primer elemento se toma en cuenta el consumo eléctrico que los equipos realizan durante su funcionamiento, como segundo elemento se detalla el costo de cada uno de los servidores, y como tercer punto se hace referencia al detalle de la administración horas/hombre.

\begin{tabular}{|c|c|c|c|c|c|c|c|c|c|}
\hline \multicolumn{10}{|c|}{ Detalle del consumo eléctrico } \\
\hline \multirow{3}{*}{ Equipos } & \multirow{3}{*}{$\mathbf{N}^{\circ}$} & \multirow{2}{*}{\multicolumn{2}{|c|}{ Potencia }} & \multicolumn{5}{|c|}{ Consumo } & \multirow{3}{*}{$\begin{array}{l}\text { KWh } \\
\$ \mathbf{0 , 0 9 5} \\
\text { valor/año }\end{array}$} \\
\hline & & & & & & & & & \\
\hline & & Vatios & $\mathbf{K V}$ & $\begin{array}{l}\text { Total } \\
\text { kw }\end{array}$ & $\begin{array}{l}\text { Uso } \\
\text { horas } \\
\text { días }\end{array}$ & $\begin{array}{l}\text { KWh } \\
\text { día }\end{array}$ & $\begin{array}{l}\text { KWh } \\
\text { mes }\end{array}$ & $\begin{array}{l}\text { KWh } \\
\text { año }\end{array}$ & \\
\hline PC/Server & 4 & 600 & $0.60 \$$ & $2.40 \$$ & $24 \mathrm{~h}$ & $57.6 \$$ & $1728 \$$ & $21024 \$$ & 1997,28 \$ \\
\hline
\end{tabular}

Tabla $N^{\circ}$ 1.1: Detalle consumo eléctrico

Elaborado por: Grupo de investigación

\begin{tabular}{llll}
\hline & \multicolumn{2}{c}{ Detalle del costo de los servidores actuales } & Costo \\
\hline Equipo & Características & Valor unitario & $3600 \$$ \\
$4 \mathrm{PC} /$ Server & Modelo Pro Desk con procesador Intel Core & $900 \$$ & \\
& i7, 4 Gb de memoria RAM, 640 GB de disco & \\
& duro, unidad de DVD RW.
\end{tabular}

TOTAL

Tabla $N^{\circ}$ 1.2: Detalle costo servidores actuales

Elaborado por: Grupo de investigación 


\begin{tabular}{lcclll}
\hline \multicolumn{7}{c}{ Detalle de la administración horas hombre para los servidores actuales } \\
\hline Equipo & Técnicos estimados & Valor hora & $\begin{array}{l}\text { Horas } \\
\text { día } 8\end{array}$ & $\begin{array}{l}\text { Total } \\
\text { mes }\end{array}$ & $\begin{array}{l}\text { Costo } \\
\text { anual }\end{array}$ \\
& & & $26 \$$ & $800 \$$ & $9600 \$$ \\
PC/Server 1 & 1 & $3,33 \$$ & $26 \$$ & $800 \$$ & $9600 \$$ \\
PC/Server 2 & 1 & $3,33 \$$ & $26 \$$ & $800 \$$ & $9600 \$$ \\
PC/Server 3 & 1 & $3,33 \$$ & $26 \$$ & $800 \$$ & $9600 \$$ \\
PC/Server 4 & 1 & $3,33 \$$ & & & $38400 \$$ \\
Total & & & &
\end{tabular}

Tabla $N^{\circ}$ 1.3: Detalle costo servidores

Elaborado por: Grupo de investigación

\section{Infraestructura Tecnológica.}

\section{Equipamiento Tecnológico Antes de la Virtualización.}

La infraestructura tecnológica que es parte de la propuesta se detalla en la siguiente tabla, cada elemento con sus respectivas características, cabe señalar que se hace referencia a los servidores utilizados anteriormente y al equipo que se implementara.

\begin{tabular}{lll}
\hline $\mathrm{N}^{\circ}$ & Descripción & \multicolumn{1}{c}{ Características } \\
\hline $\mathbf{4}$ & Computadores HP & $\begin{array}{l}\text { Modelo Pro Desk con procesador Intel Core i7, 4 Gb de memoria } \\
\text { RAM, 640 gb de disco duro, unidad de DVD RW. }\end{array}$ \\
& & Cisco serie 2900 \\
$\mathbf{1}$ & Router & D - Link \\
$\mathbf{1}$ & Switch & 48 puertos \\
$\mathbf{1}$ & RAC & Tabla $N^{\circ}$ 1.4: Detalle infraestructura tecnológica
\end{tabular}

Elaborado por: Grupo de investigación

Equipamiento Tecnológico actual para la virtualización

\begin{tabular}{lll}
\hline & Descripción & \multicolumn{1}{c}{ Características } \\
\hline $\mathbf{1}$ & Servidor HP & Xeon, 16 Gb de memoria RAM, 1 Tb en Disco duro, Hot \\
& & Swap \\
$\mathbf{1}$ & Router & Cisco serie 2900 \\
$\mathbf{1}$ & Switch & D - Link \\
$\mathbf{1}$ & RAC & 48 puertos \\
\hline
\end{tabular}

Tabla $N^{\circ}$ 1.5: Tecnología para la virtualización.

Elaborado por: Grupo de investigación.

\section{Esquema del entorno de Virtualización.}

El esquema que define la propuesta de virtualización planteada, está compuesto por hardware y software con sus respectivas características, a continuación, se detalla los diferentes elementos:

\section{Hardware.}

- Servidor HP Xeon, 16 Gb de memoria RAM, 1 Tb en Disco duro, Hot Swap, Controlador de almacenamiento HP Dynamics Smart Array B120i, controlador de red 
Ethernet NC332i,. 2 puertos de red HP Ethernet 1Gb 2 port Nc3220i, 300W de potencia de la fuente, Slot PCI-E X16:1, Slot PCI-E X8: 1.

\section{Software para Virtualización}

- Citrix XenServer 6.5

- Citrix XenCenter.

\section{Sistemas Operativos}

- Windows Server 2012 como servidor de base de datos,

- Red Hat Linux como servidor de administración.

- CentOS 6.2 como servidor de pruebas.

- CentOS 6.2 como servidor de seguridades (core).

\section{Esquema de servidores a virtualizar.}

En la siguiente gráfica se detalla los servidores que serán virtualizados, cada uno de los mismos cumplirán con su función específica para la cual fueron asignados. El elemento principal para la ejecución de la propuesta está el servidor Citrix Xen Server, el mismo que será el anfitrión para alojar a los demás sistemas operativos invitados.

Como parte de los servidores a virtualizar se encuentran los siguientes.

- Servidor de Archivos,Servidor de Base de Datos,Servidor de Control de Dominios "Active Directory", Servidor de Seguridades.

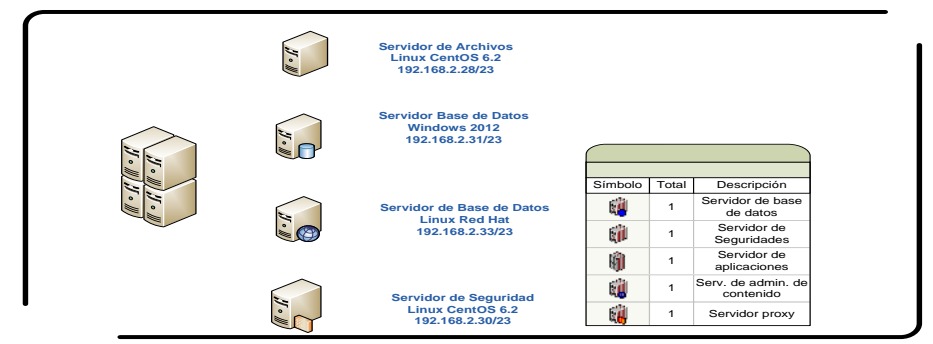

Figura $N^{\circ}$ 1.1: Esquema Servidores a Virtualizar Elaborado por: Grupo de investigación

\section{Herramienta de Virtualización Citrix XenServer. Características.}

Citrix XenServer 6.5 aumenta la velocidad, la capacidad de ampliación y el rendimiento de implementaciones de virtualización de servidores, nube y escritorios de calidad empresarial a través de mejoras de administración de redes, almacenamiento, gráficos y operaciones.

\section{Resultados.}

\section{Instalación de Citrix XenServer 6.5.}

Para el proceso de desarrollo de la propuesta existen aspectos importantes que deben ser tomados muy en cuenta con el propósito de evitar inconvenientes al momento del proceso de instalación de Citrix XenServer, a continuación se detalla cada paso para su 


\section{CiTRIX $\mid$ XenServer"m}

Figura $N^{\circ}$ 1.2.: Instalación de Citrix XenServer 6.5

Elaborado por: Grupo de investigación

\section{Instalación de Red Hat Linux Enterprise 5.}

Una vez terminado el proceso anterior, se desplegara la ventana de bienvenida del asistente de instalación del sistema operativo, en el cual se seleccionara OK, cabe señalar que este será el primer sistema operativo a instalarse en el servidor Citrix XenServer, que cumplirá la función de servidor de base de datos.

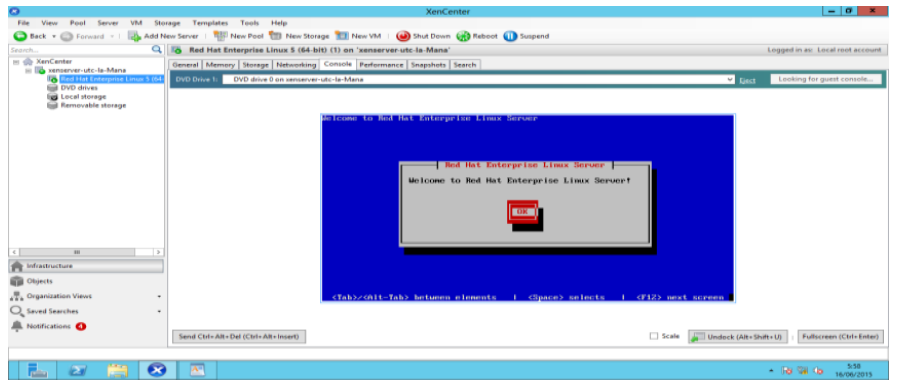

Figura $N^{\circ}$ 1.3: Pantalla de instalación de (Red Hat Enterprise Linux 5)

Elaborado por: Grupo de investigación.

\section{Instalación de Windows Server 2012.}

Para el proceso de instalación del operativo en el servidor Citrix XenServer lo que se debe hacer es el mismo procedimiento que se lo hizo para la instalación de Red Hat Enterprise Linux. Como primera opción se presenta la pantalla con el icono de Windows el cual indica que el proceso de instalación está en curso, en la cual se debe seleccionar el idioma, el formato de hora y moneda, teclado o método de entrada (español) y luego hacer clic en siguiente.

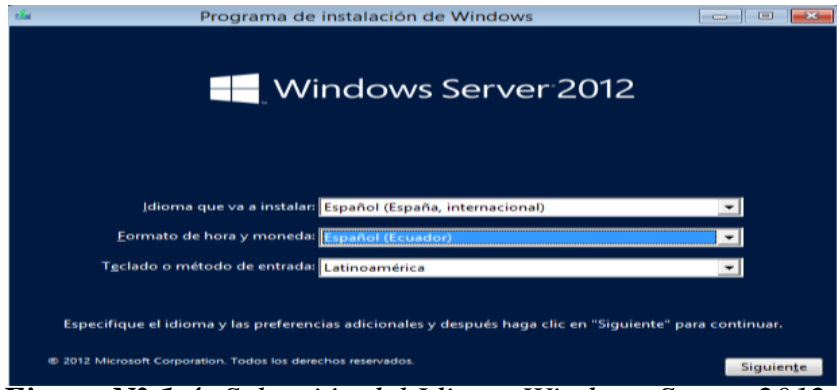

Figura $N^{\circ}$ 1.4: Selección del Idioma Windows Server 2012.

Elaborado por: Grupo de investigación. 


\section{Instalación de CentOS 6.2 .}

Para el proceso de instalación de CentOS como primer paso se introduce el dispositivo el cual contenga el sistema operativo en el servidor Citrix XenSever, una vez iniciado el proceso de instalación se mostrara la siguiente venta, en donde se seleccionara la primera opción [Install or upgrade and existing system].

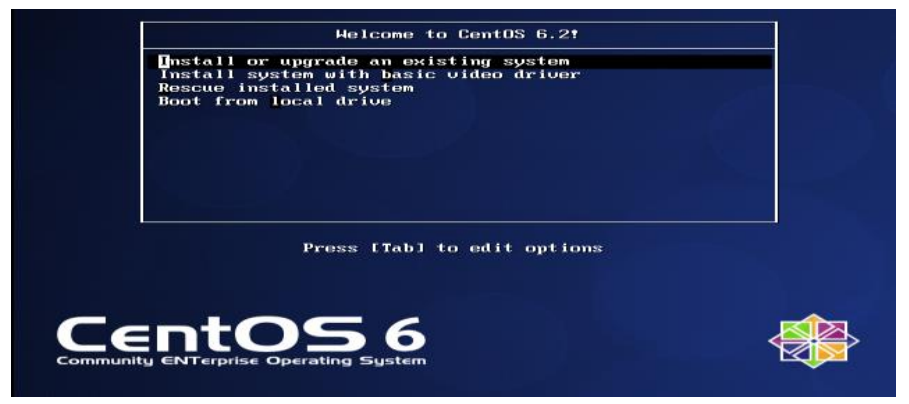

Figura $N^{\circ}$ 1.5: Instalación CentOS 6.2.

Elaborado por: Grupo de investigación.

\section{Verificación de Servicios.}

Una vez concluida la respectiva Virtualización de los servidores con la herramienta Citrix XenServer 6.5 y luego de haber terminado con la instalación de los sistemas operativos y con su respectiva configuración, se proceden hacer las pruebas necesarias para la comprobación de la hipótesis planteada. Como primer elemento que se muestra, es la conexión del cliente con el servidor de Citrix XenServer para apreciar los servidores virtualizados en el servidor principal.

\section{CiTR!X' $\mid$ XenCenter}

Figura $N^{\circ}$ 1.6: Inicio Citrix XenServer.

Elaborado por: Grupo de investigación.

Para efectuar la respectiva conexión de Citrix XenCenter con el servidor, hay que situarse en la opción XenServer y presionar clic derecho del mouse, como se muestra en la siguiente gráfica y conectarlo.

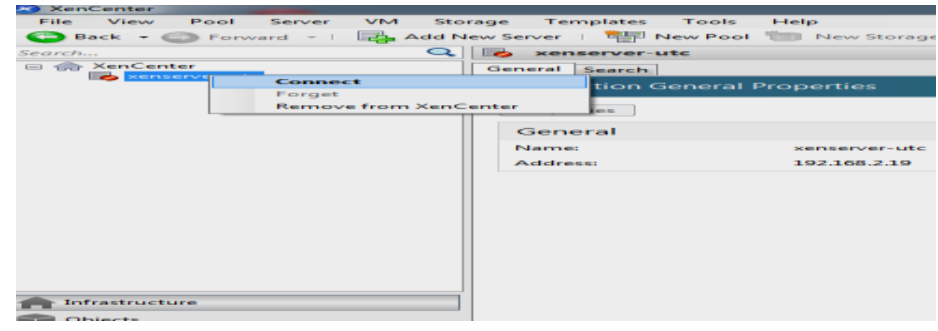


Figura $N^{\circ}$ 1.7: Conexión Citrix XenServer.

Elaborado por: Grupo de investigación.

Como siguiente paso se ingresa la dirección IP del servidor 192.168.2.19, de igual manera el nombre de usuario (root), y la respectiva contraseña (Password), y luego en conectar.

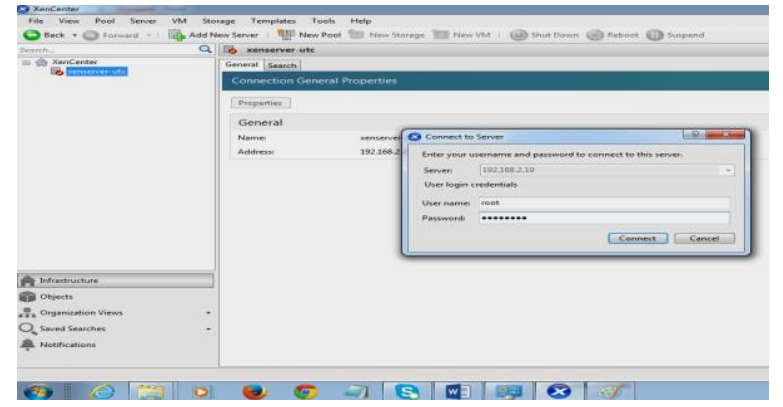

Figura $N^{\circ}$ 1.8: Conexión Citrix XenServer Ingreso de IP. Elaborado por: Grupo de investigación.

Como se puede apreciar en esta ventana se visualizan cada uno de los servidores virtualizados.

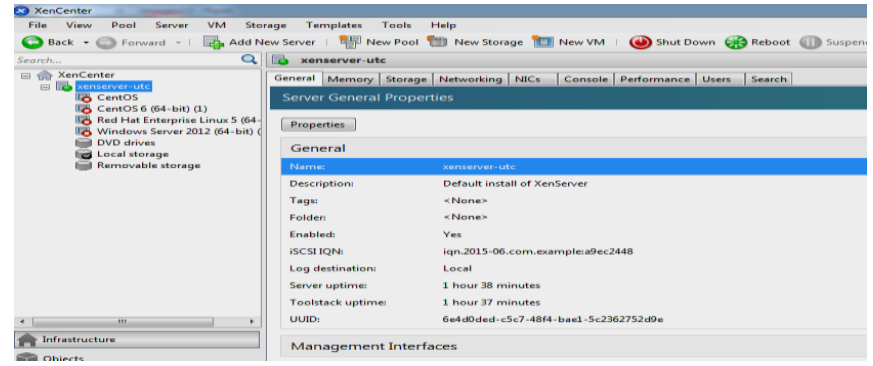

Figura $N^{\circ}$ 1.9: Visualización de Servidores Virtualizados. Elaborado por: Grupo de investigación.

Para verificar el funcionamiento y en enlace del servidor principal el cual contiene la dirección IP 192.168.2.19, de una terminal cualquiera se aplica la siguiente acción Ping 192.168.2.19. En la gráfica expuesta se aprecia que existe el respectivo enlace.

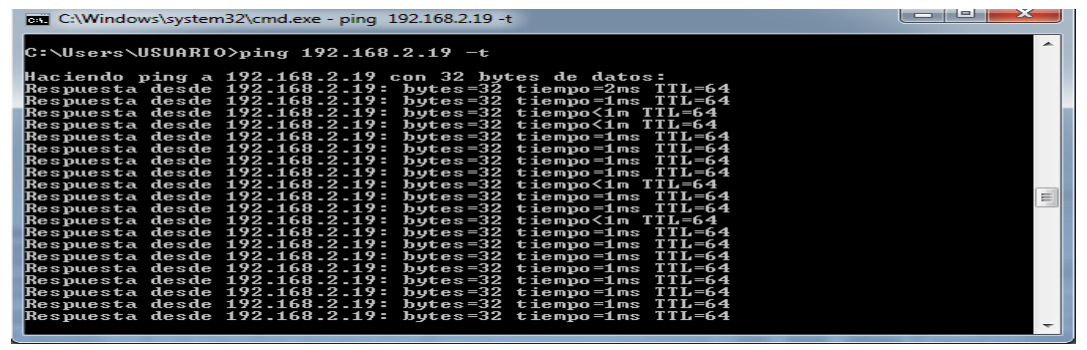

Figura $N^{\circ}$ 1.10: Comprobación de conexión.

Elaborado por: Grupo de investigación. 
El mismo procedimiento se lo realiza para el servidor de Windows 2012 Server que cumple la función de un servidor de base de datos Ping 192.168.2.31. En la gráfica expuesta se aprecia que existe la respuesta requerida. El mismo procedimiento se lo realiza para el servidor de Windows 2012 Server que cumple la función de un servidor de Active

Directory Ping 192.168.2.31. En la gráfica expuesta se aprecia que existe la respuesta requerida.

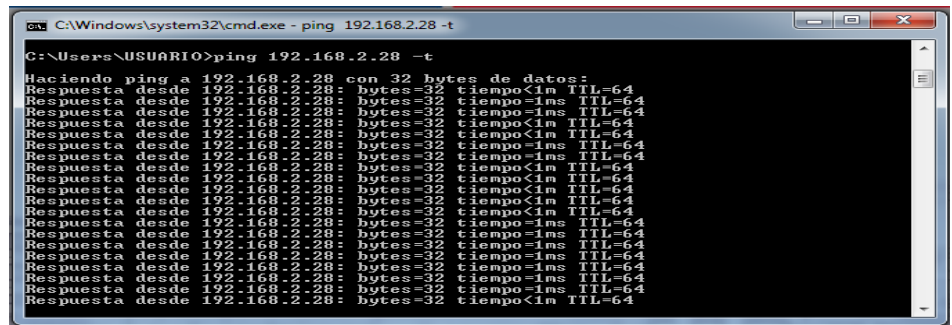

Elaborado por: Grupo de investigación.

\section{Herramienta para la Administración de los Servidores Virtualizados}

Para el proceso de administración de los servidores virtualizados, se dispone de una herramienta complementaria a Citrix XenServer, dicha herramienta de gestión unificada es Citrix XenCenter, para lo cual es necesaria su instalación.

\section{CiTR!X: $\mid$ XenCenter}

Figura $N^{\circ}$ 1.12: Instalación de Citrix XenCenter. Elaborado por: Grupo de investigación.

Luego hay que seleccionar la carpeta de destino de la instalación, en esta ocasión no se realiza ningún cambio y luego seleccionar "Next" para continuar.

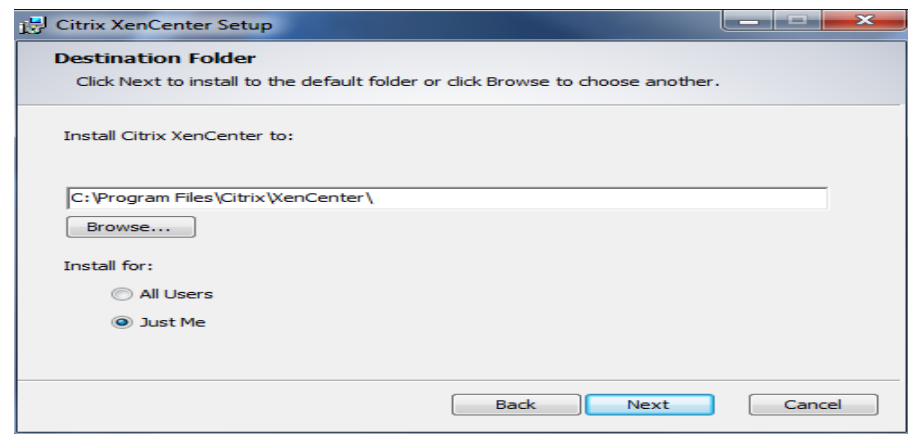

Figura $N^{\circ}$ 1.13: Instalación de Citrix XenCenter Elaborado por: Grupo de investigación 
Con el Servidor XenServer en producción, se procede a iniciar la herramienta de gestión unificada XenCenter, se lo puede hacer desde cualquier equipo de la red con acceso al servidor, se podrá realizar la comprobación y analizar estadísticas de uso y rendimiento de los recursos del servidor, de igual manera se puede realizar las tareas de la administración del XenServer y de los servidores virtualizados independientemente.

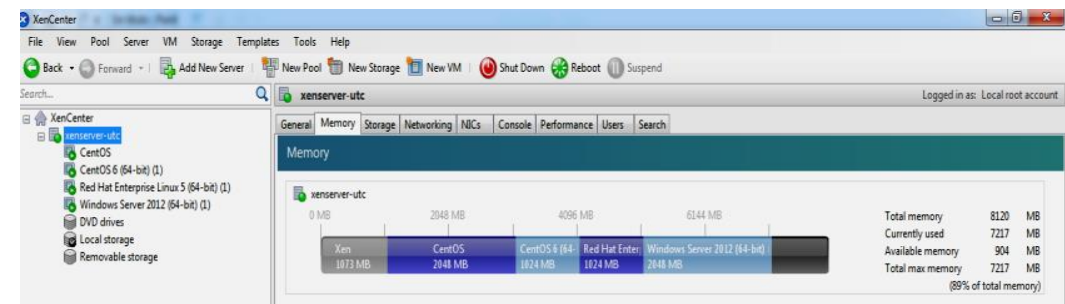

Figura $N^{\circ}$ 1.14: Verificación de memoria utilizada.

Elaborado por: Grupo de investigación.

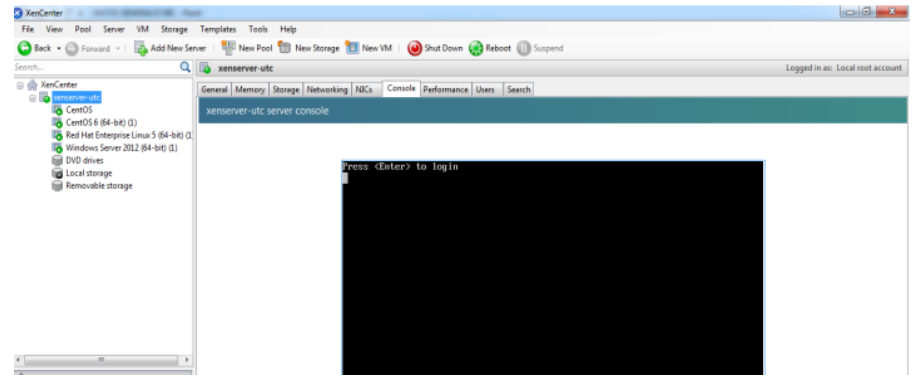

Figura $N^{\circ}$ 1.15: Consola de Citrix XenServer.

Elaborado por: Grupo de investigación.

Por ejemplo, para poder administrar cualquier servidor virtualizado es necesario iniciar su funcionamiento, al situarse en la pestaña "Perfomance", se podrá apreciar las estadísticas de como: uso del procesador, memoria, red y disco, desde la opción "Perfomance" se aprecian los recursos individuales que hacen uso cada VM (Máquina Virtual).

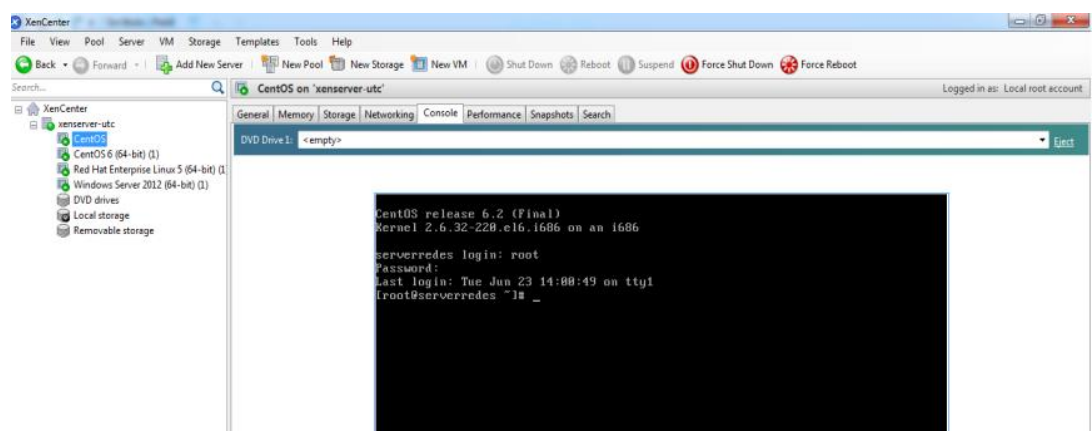

Figura $N^{\circ}$ 1.16: Consola de CentOS.

Elaborado por: Grupo de investigación.

Mediante la pestaña "Search" se puede apreciar las estadísticas y opciones de rendimiento de cada VM (Máquina Virtual), para ello deben estar encendidas. 


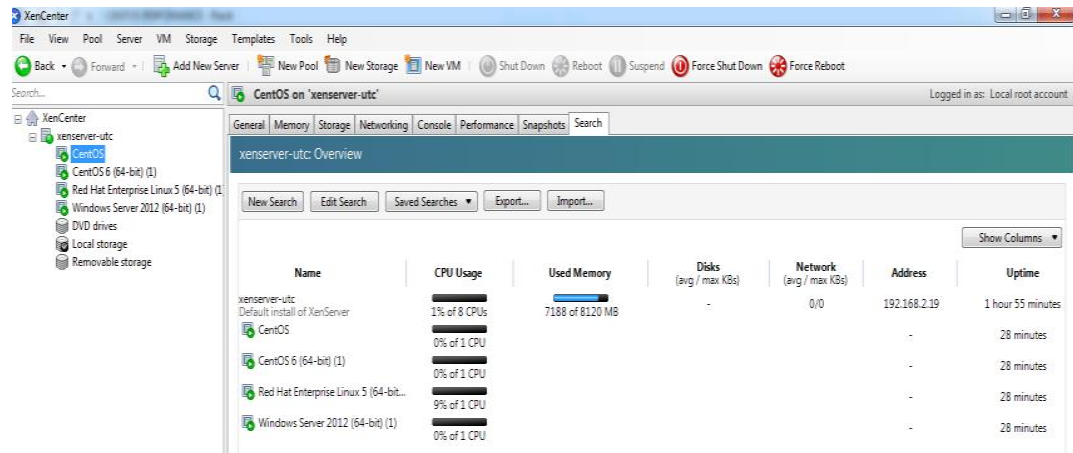

Figura $N^{\circ}$ 1.18: Buscador de uso de memoria Servidor Virtualizado . Elaborado por: Grupo de investigación.

En esta venta se puede apreciar el uso del CPU del servidor virtualizado.

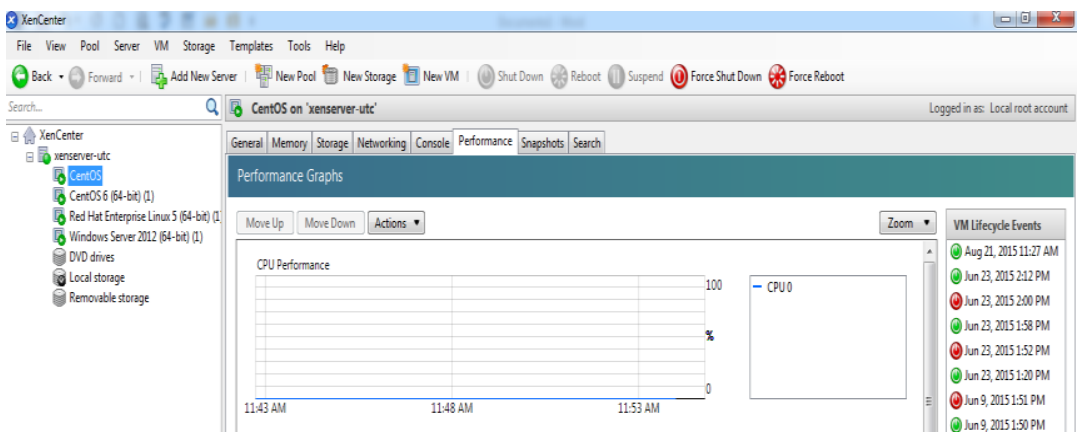

Figura $N^{\circ}$ 1.19: Uso de CPU Servidor CentOS.

Elaborado por: Grupo de investigación.

En la pestaña "perfomance" se puede apreciar las estadísticas y opciones de rendimiento de cada VM (Máquina Virtual), es así que se pude apreciar el uso del disco, en este caso del servidor CentOS.

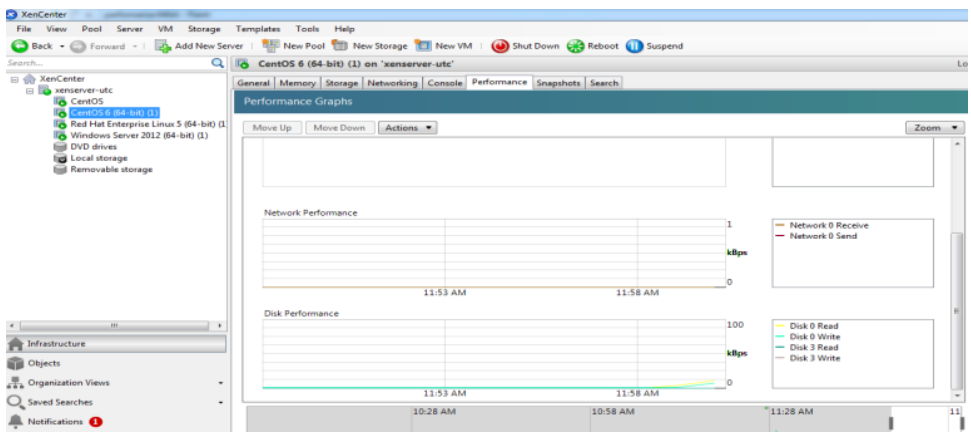

Figura $N^{\circ}$ 1.20: Uso de CPU Servidor CentOS .

Elaborado por: Grupo de investigación. 


\section{Implementación.}

Luego de haber concluido con las etapas de desarrollo y de pruebas, finalmente se hace necesario establecer la implementación respectiva, es así que en el siguiente diagrama se puede apreciar el producto final de la propuesta.

El esquema de virtualización aplicado en el laboratorio de redes se estableció en base a las necesidades de la Institución, la investigación desarrollada cumple con los objetivos que inicialmente se plantearon, en tal sentido existe la satisfacción del personal que se encarga de la administración de los laboratorios, ya que fue en aquella área en donde se implementó y presta todas las garantías del caso, en la presente imagen se muestra el esquema final de virtualización de servidores, cada uno ellos cumplen con su función a la cual fueron asignados.

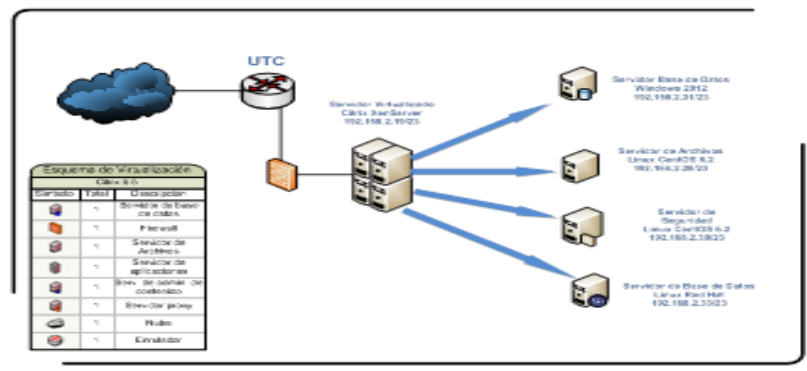

Figura $N^{\circ}$ 1.21: Esquema final.

Elaborado por: Grupo de investigación.

Es así como finalmente se muestran los servidores consolidados, para ponerlos en funcionamiento se debe seleccionar la opción STAR en cada uno de ellos, es importante conocer que con esta herramienta Citrix XenServer y Citrix XenCenter, se puede trabajar con los cuatro servidores de manera simultánea sin tener que suspender el funcionamiento de ninguno de los otros servidores, cabe señalar que la herramienta encargada de la Administración de los servidores virtualizados es la herramienta Citrix XenCenter.

En esta sección se muestran cada uno de los servidores en funcionamiento.

\section{CentOS (Servidor de Archivos).}

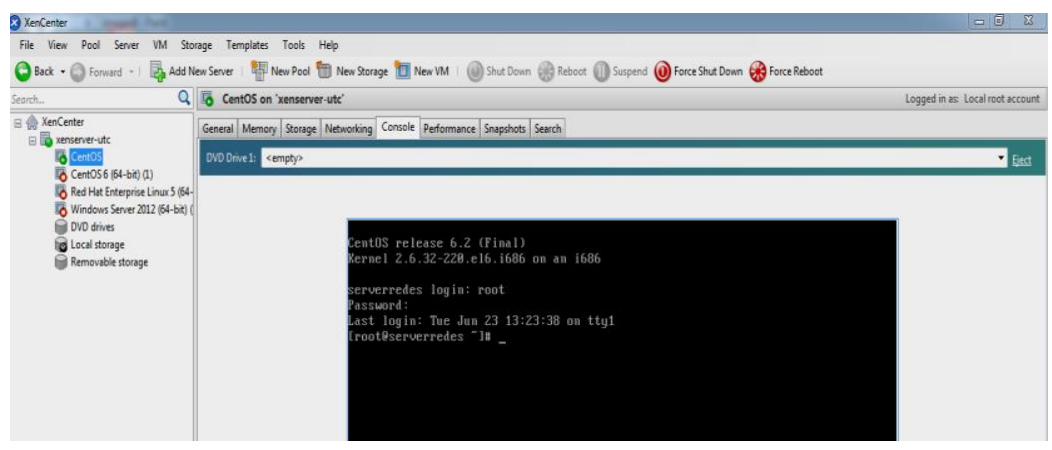

Figura $N^{\circ}$ 1.22: Inicio Servidor CentOS

Elaborado por: Grupo de investigación 
CentOS (64 bits (1)) (Servidor de Seguridades).

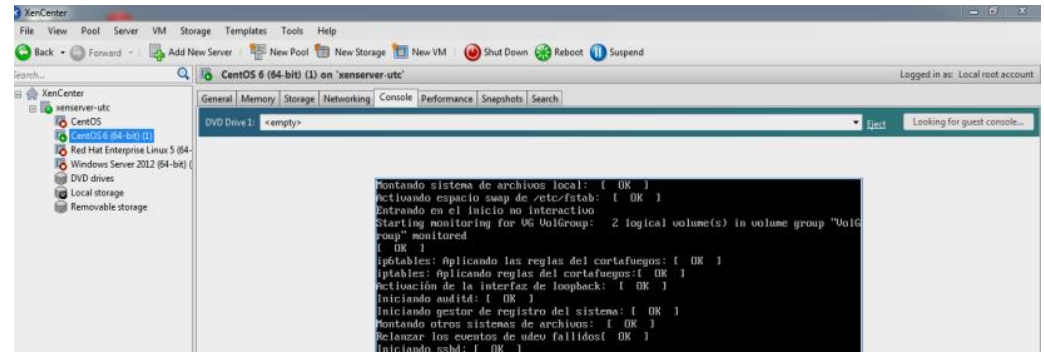

Figura $N^{\circ}$ 1.28: Inicio Servidor CentOS (64 bits).

Elaborado por: Grupo de investigación.

\section{Red Hat Enterprise Linux (Servidor de Base de Datos).}

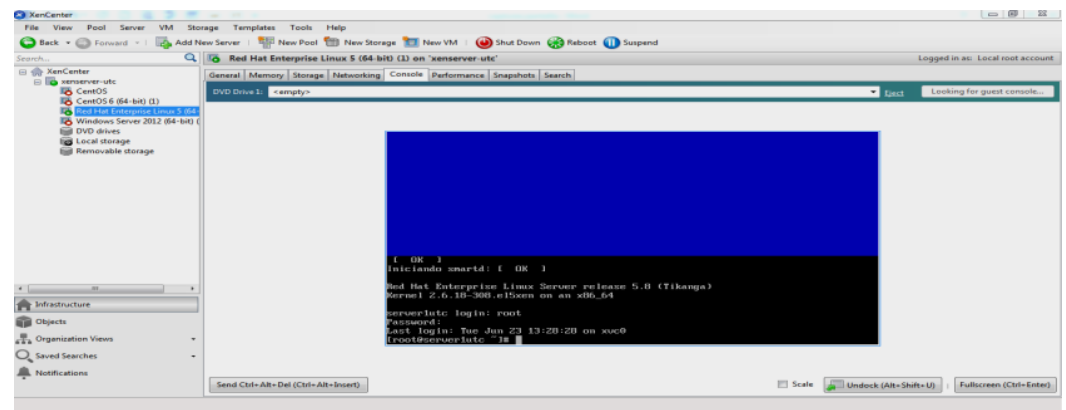

Figura $N^{\circ}$ 3.29: Inicio Servidor Linux.

Elaborado por: Grupo de investigación.

\section{Windows Server 2012 (Servidor Active Directory).}

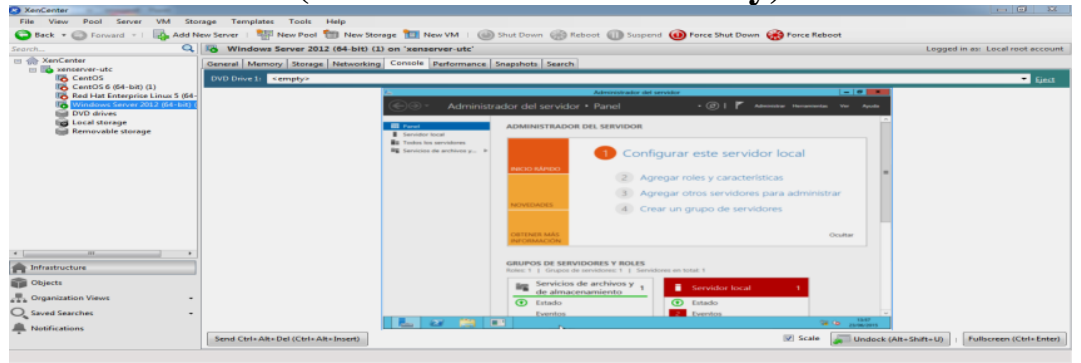

Figura $N^{\circ}$ 1.30: Inicio Servidor Windows 2012 Server.

Elaborado por: Grupo de investigación.

\section{Servidores consolidados en funcionamiento.}

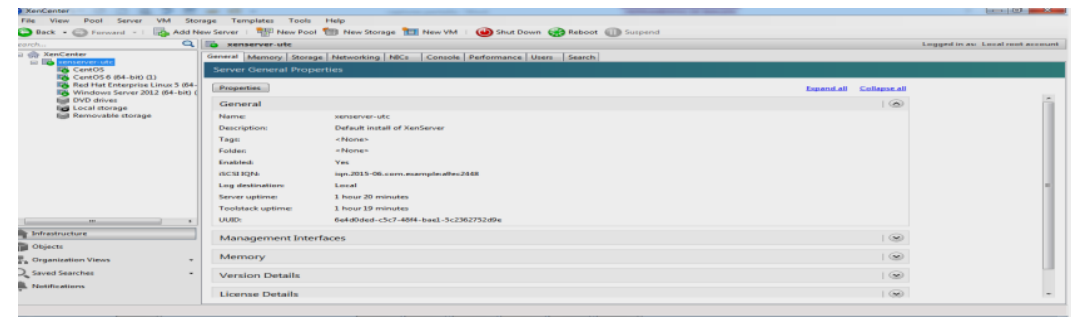

Figura $N^{\circ}$ 3.31: Servidores Consolidados.

Elaborado por: Grupo de investigación. 


\section{Tablas comparativas de optimización de recursos.}

En las siguientes tablas se desarrolla el respectivo análisis comparativo sobre el cómo se optimizaron los recursos en los laboratorios de la institución, dicha comparación se lo estableció en base al número de servidores que anteriormente funcionaban de forma individual, para lograr con el objetivo propuesto.

\begin{tabular}{cccc}
\hline \multicolumn{3}{c}{ Detalle del costo de los servidores actuales } \\
\hline Equipo & $\begin{array}{c}\text { Características } \\
\text { Modelo Pro Desk con } \\
\text { procesador Intel Core i7, 4 Gb } \\
\text { de memoria RAM, 640 GB de } \\
\text { disco duro, unidad de DVD }\end{array}$ & Valor unitario & Costo \\
RW. & $900 \$$ & $3600 \$$ \\
TOTAL & $\begin{array}{l}\text { RWer } \\
\text { TOT }\end{array}$ & $3600 \$$ \\
\hline
\end{tabular}

Tabla $N^{\circ}$ 1.5: Detalle costo servidores

Elaborado por: Grupo de investigación

Equipo Actual

\begin{tabular}{cccc}
\hline Nombre & Características & Valor Unitario & Costo \\
Servidor HP & 16Ram,2Terabyte en & $2100 \$$ & $2100 \$$ \\
\hline
\end{tabular}

Tabla $N^{\circ}$ 1.6: Detalle costo equipo actual

Elaborado por: Grupo de investigación

Es evidente que, con la virtualización de servidores, en base a las tablas comparativas se puede apreciar que existe una reducción importante en los costos de los equipos, es así que se pude establecer un ahorro económico para la institución, como siguiente aspecto se hace la comparación detallada sobre el consumo eléctrico que genera cada uno de los servidores anteriores, con el consumo que lo realiza ya una vez finalizada la implementación

\section{Detalle del consumo eléctrico}

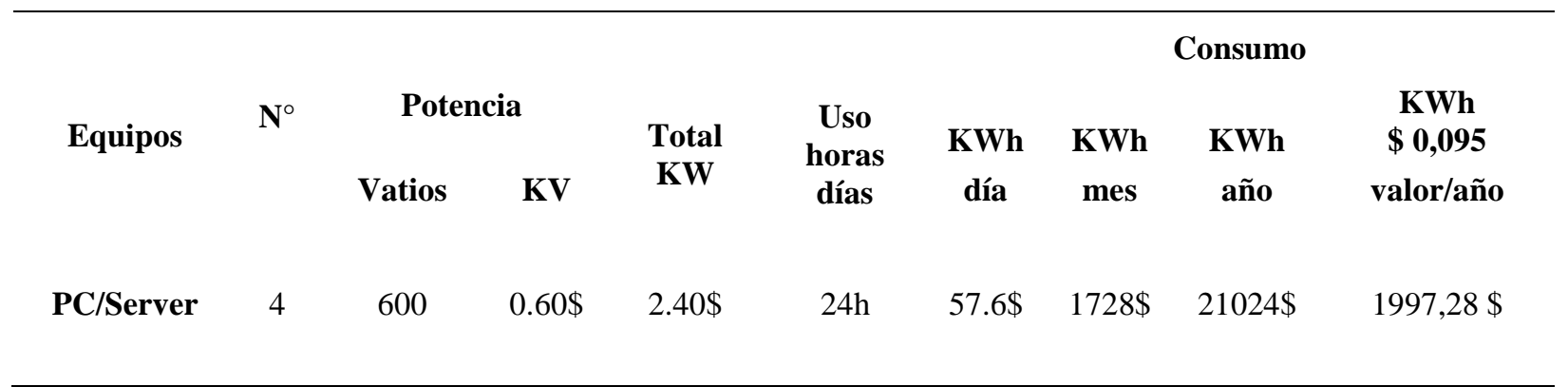

Tabla $N^{\circ}$ 1.7: Detalle consumo eléctrico antes de virtualizar.

Elaborado por: Grupo de investigación. 
Detalle del consumo eléctrico servidor CITRIX XenServer

\begin{tabular}{|c|c|c|c|c|c|c|c|c|c|}
\hline \multirow{3}{*}{ Equipos } & \multirow{3}{*}{$\mathbf{N}^{\circ}$} & & & & & \multicolumn{4}{|c|}{ Consumo } \\
\hline & & \multicolumn{2}{|c|}{ Potencia } & \multirow{2}{*}{$\begin{array}{l}\text { Total } \\
\text { KW }\end{array}$} & \multirow{2}{*}{$\begin{array}{l}\text { Uso } \\
\text { horas } \\
\text { días }\end{array}$} & & & & KWh \\
\hline & & Vatios & KV & & & día & mes & año & valor/año \\
\hline $\begin{array}{c}\text { Xeon, } 16 \mathrm{~Gb} \text { de } \\
\text { memoria RAM, } 1 \text { Tb en } \\
\text { Disco duro, Hot Swap }\end{array}$ & 1 & 300 & $0.30 \$$ & $1.20 \$$ & $24 \mathrm{~h}$ & $28.8 \$$ & $864 \$$ & $10512 \$$ & $998,64 \$$ \\
\hline
\end{tabular}

Tabla $N^{\circ}$ 1.8: Detalle consumo eléctrico después de virtualizar.

Elaborado por: Grupo de investigación.

De acuerdo a la comparación sobre el consumo eléctrico, es muy importante notar la diferencia que existe entre las dos alternativas, sin duda alguna antes de aplicar la propuesta planteada se puede apreciar que existe un consumo considerable del recurso energético, mientras tanto que luego de la implementación de la virtualización de servidores se puede apreciar una diferencia marcada sobre el consumo eléctrico.

10

\begin{tabular}{cccccc}
\hline Equipo & $\begin{array}{c}\text { Técnicos } \\
\text { Estimados }\end{array}$ & Valor Hora & $\begin{array}{c}\text { Horas } \\
\text { día } 8\end{array}$ & $\begin{array}{c}\text { Total } \\
\text { mes }\end{array}$ & $\begin{array}{c}\text { Costo } \\
\text { Anual }\end{array}$ \\
PC/Server 1 & 1 & & $26 \$$ & $800 \$$ & $9600 \$$ \\
PC/Server 2 & 1 & $3,33 \$$ & $26 \$$ & $800 \$$ & $9600 \$$ \\
PC/Server 3 & 1 & $3,33 \$$ & $26 \$$ & $800 \$$ & $9600 \$$ \\
PC/Server 4 & 1 & $3,33 \$$ & $26 \$$ & $800 \$$ & $9600 \$$ \\
TOTAL & & & & & $38400 \$$ \\
\hline
\end{tabular}

Tabla $N^{\circ}$ 1.9: Detalle administración horas hombre antes de virtualizar.

Elaborado por: Grupo de investigación.

\begin{tabular}{|c|c|c|c|c|c|}
\hline \multicolumn{6}{|c|}{ Detalle de la Administración horas hombre para Citrix XenServer } \\
\hline Equipo & Técnicos Estimados & Valor hora & $\begin{array}{c}\text { Horas } \\
\text { día } 8\end{array}$ & $\begin{array}{c}\text { Total } \\
\text { mes }\end{array}$ & $\begin{array}{l}\text { Costo } \\
\text { anual }\end{array}$ \\
\hline \multirow{2}{*}{$\begin{array}{c}\text { Xeon, } 16 \text { Gb de } \\
\text { memoria RAM, } \\
1 \text { Tb en Disco } \\
\text { duro, Hot Swap }\end{array}$} & 1 & $3,33 \$$ & $26 \$$ & $800 \$$ & $9600 \$$ \\
\hline & 1 & $3,33 \$$ & $26 \$$ & $800 \$$ & $9600 \$$ \\
\hline TOTAL & & & & & $19200 \$$ \\
\hline
\end{tabular}

Tabla $N^{\circ}$ 1.10: Detalle administración horas hombre después de virtualizar.

Elaborado por: Grupo de investigación. 
El recurso económico sin duda alguna es el más importante ya que el mismo es el factor con el cual las organizaciones pueden ejecutar sus procesos, es así que es transcendental identificar en el análisis de este recurso que existe un ahorro proyectado al solo contar con dos técnicos que puedan cubrir el manejo adecuado de los servidores virtualizados.

\section{Conclusiones.}

- Mediante el estudio de la virtualización de servidores, se establece que la misma son soluciones completamente adaptables a los entornos de las instituciones, ya que satisface la necesidad y requerimientos.

- La herramienta que se establece como referente para la virtualización es Citrix XenServer por ser una herramienta gratuita y por su entorno amigable.

- Al implementar la virtualización el ahorro en los recursos tecnológicos, es evidente en aspectos como: ahorro en el consumo eléctrico, así también en el costo de los equipos y la administración de los servidores.

- Las tecnologías de virtualización son herramientas tecnológicas que ayudan a la consolidación de los recursos de software dentro de un mismo elemento, al igual que las tecnologías verdes su propósito es el de favorecer a la optimización de recursos

- La optimización de recursos es un elemento muy importante ya la misma tiene como objetivo la utilización de los recursos de una manera óptima y eficaz para el buen desarrollo de las organizaciones

\section{Referencias bibliográficas.}

AGUILERA, P. (2011). Seguridad Informática. Madrid: Editex.

BERMEJO, J. M. (2014). Innovación continua en el exito empresarial. Madrid, España: UNED.

CITRIX. (2013). lac.citrix.com. (lac.citrix.com/) Recuperado el 19 de 06 de 2015, de lac.citrix.com/: https://lac.citrix.com/products/xenserver/tech-info.html

GALLEGO, J. (2014). Operaciones Auxiliares para la configuracion y la Explotación. Madrid: Editex.

GOYANES, L. (2012). Computacion en la Nube. Estrategias de Cloud Computing en las Empresas (Primera ed.). Mexico, Mexico: Alfaomega.

JIMENEZ, D. (2011). Cloud Computing: Retos y Oportunidades. Madrid, España: Fundación IDEAS.

JOYANES, L. (2012). Computacion en la nube Estrategias de Cloud Computing en las Empresas (Primera ed.). México, México: Alfaomega.

KHAN, S. (2013). Studies in Computational Intelligent/ Evolutionary Based Solutions for Green Computing. Verlag: Springer. 
NIÑO, J. (2011). Sistemas Operativos Monopuestos. Madrid: Editex.

RAYA, J. M. (2010). Guia de campo Maquinas Virtuales (Primera ed.). México, México: Alfaomega.

RAYA, L. (2010). Maquinas Virtuales (Primera ed.). Mexico: Alfaomega.

ROJAS, M. (19 de Febrero de 2013). Recusos Tecnologicos. Recuperado el 03 de julio de 2015, de http://usosdelatecnologiaenelaula.blogspot.com/2013/02/recursostecnologicos-definicion.html

SANCHEZ, J. (Abril de 2008). EUMED.NET/ ENCICLOPEDIA VIRTUAL. (EUMED.NET) Recuperado el 01 de JUNIO de 2015, de EUMED.NET/ ENCICLOPEDIA VIRTUAL: http://www.eumed.net/libros-gratis/2008b/390/index.htm

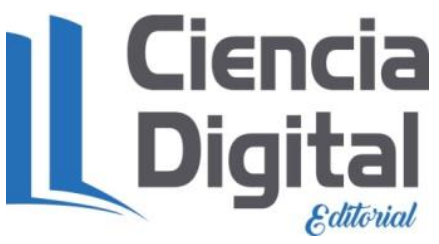


Para citar el artículo indexado.

Jácome D., Núñez J.,Velastegui E., Navas M., \& Vásquez P (2018). La virtualización de servidores como una herramienta para la optimización de recursos. Revista electrónica Ciencia Digital 2(2), 280-305. Recuperado desde:

http://www.cienciadigital.org/index.php/CIENCIADIGITAL/article/view/67/Publicacion1 $\% 2 \mathrm{CNo} 2 \% 2 \mathrm{CVol1}$

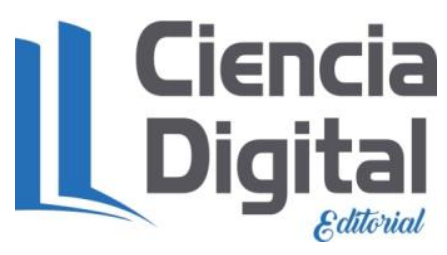

El artículo que se publica es de exclusiva responsabilidad de los autores y no necesariamente reflejan el pensamiento de la Revista Ciencia Digital.

El articulo queda en propiedad de la revista y, por tanto, su publicación parcial y/o total en otro medio tiene que ser autorizado por el director de la Revista Ciencia Digital.
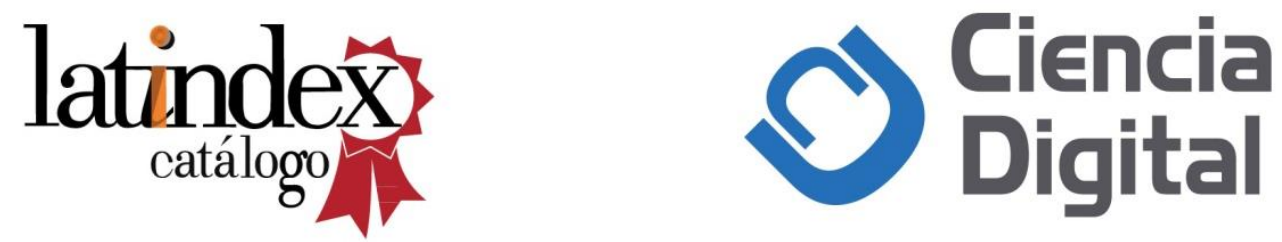